\title{
ON THE MAXIMUM MODULUS AND THE MAXIMUM TERM OF AN ENTIRE DIRICHLET SERIES
}

\author{
A. G. AZPEITIA ${ }^{1}$
}

1. We consider the Dirichlet series

$$
f(s)=\sum_{n=0}^{\infty} a_{n} e^{\lambda_{n} s} \quad\left(s=\sigma+i t, \lambda_{0}=0, \lambda_{n}<\lambda_{n+1} \rightarrow \infty\right)
$$

which we assume to be absolutely convergent for all $s$. We define the maximum modulus over a vertical line as

$$
M(\sigma)=\underset{-\infty<i<\infty}{\text { l.u.b. }}|f(\sigma+i t)|
$$

and the maximum term as

$$
m(\sigma)=\max _{n \geq 0}\left|a_{n} e^{\lambda_{n}(\sigma+i t)}\right| .
$$

Finally, the index function $n(\sigma)$ is given by

$$
n(\sigma)=\max _{n \geqq 0}\left\{n|m(\sigma)=| a_{n} \mid e^{\lambda_{n} \sigma}\right\} .
$$

The function $\log M(\sigma)$ is divergent to $+\infty$ for $\sigma \rightarrow+\infty[1, \mathrm{p} .77]$ and convex $[2$, p. 240]. By analogy with the case of Taylor series, $[3, \mathrm{pp} .37-38]$ and making use of the absolute convergence of (1), it is an easy matter to show that the same properties hold for $m(\sigma)$, and that $n(\sigma)$ is a step function divergent to $+\infty$ for $\sigma \rightarrow+\infty$, continuous from the right for all $\sigma$, having only a finite number of discontinuities in every infinite interval of the form $(-\infty, b)$, and related to $m(\sigma)$ by the formula, valid for all $\sigma$ and $\sigma^{\prime}$,

$$
\log m(\sigma)=\log m\left(\sigma^{\prime}\right)+\int_{\sigma^{\prime}}^{\sigma} \lambda_{n(x)} d x .
$$

It is also known $[4$, p. 170 , Theorem IX] that

$$
m(\sigma) \leqq M(\sigma) \text {. }
$$

If we denote by $n_{0}$ the index of the first $a_{n}$ which is not zero and by $\left\{\sigma_{i}\right\}(i=0,1,2, \cdots)$ the monotonic increasing sequence of discontinuities of $n(\sigma)$ (taking $\sigma_{0}=-\infty$ for the sake of completeness) then

Received by the editors November 2, 1960.

1 This work is part of research supported by a grant from the National Science Foundation (NSF-G-14142). 
the sequence of indices defined by $n_{i}=n\left(\sigma_{i}\right)(i=0,1,2, \ldots)$ is such that

$$
n(\sigma)=n_{i} \quad \text { for } \sigma_{i} \leqq \sigma<\sigma_{i+1} .
$$

2. Since the assumption that the series (1) is absolutely convergent for all $s$ implies $\log \left|a_{n}\right| / \lambda_{n} \rightarrow-\infty$, it is possible to construct a Newton-Hadamard polygon for the sequence of points $P_{n}$ with coordinates $\lambda=\lambda_{n}, y=-\log \left|a_{n}\right|$ in a cartesian plane $(\lambda, y)$. The sequence of vertices of such polygon corresponds to the sequence of indices $n_{i}$ and has the property that the slope of the side $P_{n_{i-1}} P_{n_{i}}$ is equal to $\sigma_{i}$. The proofs of these facts are entirely similar to the ones in the case of Taylor series $[5$, p. $6 ; 6$, p. 274].

3. Let us now assume that three strictly increasing, divergent sequences $\left\{n_{i}\right\},\left\{\sigma_{i}\right\}$ and $\left\{\lambda_{n}\right\}\left(i, n=0,1,2, \cdots ; \lambda_{0}=0, \sigma_{0}=-\infty\right)$ with the $n_{i}$ being non-negative integers, and the $\lambda$ 's such that

$$
\infty \geqq \liminf _{n=\infty} \lambda_{n} / \log n=\gamma>0
$$

are given, and let us consider the series

$$
\sum_{n=0}^{\infty} A_{n} e^{\lambda_{n} s}
$$

where the $A_{n}$ are defined in the following way: $A_{n}=0$ for $n<n_{0}$, $A_{n_{0}}=a, a$ being any arbitrary real constant, and $A_{n}$ $=A_{n_{i-1}} \exp \left[-\left(\lambda_{n}-\lambda_{n_{i-1}}\right) \sigma_{i}\right]$ for $n_{i-1}<n \leqq n_{i}$. Since the sequence $\left\{-\log \left(A_{n} / A_{n-1}\right) /\left(\lambda_{n}-\lambda_{n-1}\right)\right\}$ is obviously monotonic and divergent, it follows that the sequence of points $Q_{n}$ of the plane $(\lambda, y)$ having coordinates $\lambda=\lambda_{n}, y=-\log A_{n}$ defines a Newton-Hadamard polygon whose vertices are $Q_{n_{i}}$ and whose sides contain all the points $Q_{n}$. This implies that the $m(\sigma)$ function corresponding to the series (6) is given by $m(\sigma)=A_{n_{i}} \exp \left(\lambda_{n_{i}} \sigma\right)$ for $\sigma_{i} \leqq \sigma<\sigma_{i+1}$. Finally, we can prove that (6) is absolutely convergent for all $\sigma$ in the following way: For every given $s=\sigma+i$ we can select three numbers $\gamma_{1}, \gamma_{2}, K$ such that $0<\gamma_{1}<\gamma_{2}<\gamma \leqq \infty$ and $K>\sigma+\left(1 / \gamma_{1}\right)$. Then by (5) we have that from some $n$ on, $\lambda_{n}>\gamma_{2} \log n$. Also, since $\sigma_{i} \uparrow \infty$, it follows that $\log A_{n}$ $<-K \lambda_{n}$ for all $n$ large enough. Therefore, there exists some integer $N$ such that

$$
\begin{aligned}
\sum_{n=N}^{\infty}\left|A_{n} e^{\lambda_{n} s}\right| & <\sum_{n=N}^{\infty} e^{(\sigma-K) \lambda_{n}}<\sum_{n=N}^{\infty} e^{-\left(1 / \gamma_{1}\right) \lambda_{n}}<\sum_{n=N}^{\infty} e^{-\left(\gamma_{2} / \gamma_{1}\right) \log n} \\
& =\sum_{n=N}^{\infty} n^{-\left(\gamma_{2} / \gamma_{1}\right)}<\infty .
\end{aligned}
$$


Consequently, the series (6) defines an entire function $F(s)$ and if the sequences $\left\{n_{i}\right\},\left\{\sigma_{i}\right\},\left\{\lambda_{n}\right\}$ are the ones corresponding to the series (1), and we take $a=\left|a_{n_{0}}\right|$, then both series (1) and (6) have the same index function $n(\sigma)$ and the same maximum term $m(\sigma)$ given by (2). In addition, their coefficients satisfy the relations $\left|a_{n}\right| \leqq A_{n}$ for all $n=0,1,2, \cdots$ and $\left|a_{n_{i}}\right|=A_{n_{i}}$ for all $i=0,1,2, \cdots$, and finally

$$
M(\sigma) \leqq \max _{-\infty<t<\infty}|F(\sigma+i t)|=F(\sigma)
$$

holds for all $\sigma$.

4. We now consider the problem of the asymptotic equivalence of the functions $\log M(\sigma)$ and $\log m(\sigma)$. If $\lim \inf _{n=\infty} \log \lambda_{n} / \log n>0$, (which implies (5)), holds, and either

$$
\lim \sup \log \log M(\sigma) / \sigma<\infty
$$

or, what is equivalent, [7, p. 109, Theorem (2.8)],

$$
\limsup _{\sigma=\infty} \log \log m(\sigma) / \sigma<\infty
$$

that is to say, if the function is of finite Ritt order [1, p. 77], then $\log M(\sigma)$ and $\log m(\sigma)$ are asymptotically equivalent, [6, p. 265, Theorem 5].

With no restriction on the order but assuming

$$
\liminf _{n=\infty}\left(\lambda_{n}-\lambda_{n-1}\right)=\beta>0
$$

we will establish sufficient conditions for $\log M(\sigma)$ and $\log m(\sigma)$ to be asymptotically equivalent. We begin by proving the following theorem which generalises the inequality of Valiron $[8, \mathrm{p} .32$, Theorem 11].

THEOREM 1. If the series (1) is absolutely convergent for all $s$ and (8) holds, then for every $\beta_{1}<\beta$ and all $\sigma>\sigma\left(\beta_{1}\right)$

$$
m(\sigma) \leqq M(\sigma) \leqq\left[1+n(\sigma+\delta(\sigma))+\frac{1}{e^{\beta_{1} \delta(\sigma)}-1}\right] m(\sigma)
$$

where $\delta(\sigma)$ is any positive, but otherwise arbitrary, function of $\sigma$.

Proof. For every $\sigma$, we define the integer $k$ by the equality $n_{k}=n(\sigma+\delta(\sigma))$, and for every index $m>n_{k}$ we define the integer $p$ by $n_{p} \geqq m>n_{p-1}$. It follows that $\sigma+\delta(\sigma)<\sigma_{k+1}$ and $k<p$ and therefore, since (8) implies (5) and the conclusions of the previous section are valid, we have 


$$
\begin{aligned}
& A_{m} \exp \left(\lambda_{m} \sigma\right)=a \exp \left[-\left(\lambda_{n_{1}}-\lambda_{n_{0}}\right) \sigma_{1}-\cdots-\left(\lambda_{n_{k}}-\lambda_{n_{k-1}}\right) \sigma_{k}-\cdots\right. \\
& \left.-\left(\lambda_{m}-\lambda_{n_{p-1}}\right) \sigma_{p}+\lambda_{m} \sigma\right] \\
& =A_{n_{k}} \exp \left(\lambda_{n_{k}} \sigma\right) \exp \left[-\left(\lambda_{n_{k+1}}-\lambda_{n_{k}}\right) \sigma_{k+1}-\cdots\right. \\
& \left.-\left(\lambda_{m}-\lambda_{n_{p-1}}\right) \sigma_{p}+\left(\lambda_{m}-\lambda_{n_{k}}\right) \sigma\right] \\
& <m(\sigma) \exp \left[\left(\lambda_{m}-\lambda_{n_{k}}\right)\left(\sigma-\sigma_{k+1}\right)\right] .
\end{aligned}
$$

Now, according to (7), we have for all $\sigma>\sigma\left(\beta_{1}\right)$

$$
\begin{aligned}
M(\sigma) & \leqq F(\sigma)=\sum_{m=0}^{n_{k}} A_{m} \exp \left(\lambda_{m} \sigma\right)+\sum_{m=n_{k}+1}^{\infty} A_{m} \exp \left(\lambda_{m} \sigma\right) \\
& <\left\{1+n_{k}+\sum_{m=n_{k}+1}^{\infty} \exp \left[\left(\lambda_{m}-\lambda_{n_{k}}\right)\left(\sigma-\sigma_{k+1}\right)\right]\right\} m(\sigma) \\
& \leqq\left\{1+n_{k}+\sum_{m=n_{k}+1}^{\infty} \exp \left[\left(m-n_{k}\right) \beta_{1}\left(\sigma-\sigma_{k+1}\right)\right]\right\} m(\sigma) \\
& =\left(1+n_{k}+\exp \left[\beta_{1}\left(\sigma-\sigma_{k+1}\right)\right] /\left\{1-\exp \left[\beta_{1}\left(\sigma-\sigma_{k+1}\right)\right]\right\}\right) m(\sigma) \\
& \leqq\left\{1+n(\sigma+\delta(\sigma))+1 /\left[\exp \left(\beta_{1} \delta(\sigma)\right)-1\right]\right\} m(\sigma)
\end{aligned}
$$

and the theorem is proved.

Finally, we will demonstrate the following result:

THEOREM 2. Under the same assumptions of Theorem 1, if there is a positive constant $\alpha$ such that either

$$
\lim _{\sigma=\infty} \log \log m(\sigma+\alpha) / \log m(\sigma)=0
$$

or

$$
\lim _{\sigma=\infty} \log \log M(\sigma+\alpha) / \log M(\sigma)=0
$$

then

$$
\lim _{\sigma=\infty} \log M(\sigma) / \log m(\sigma)=1 .
$$

Proof. By (2) and (8), we have, for all $\sigma$ large enough and any $\alpha>0$ and some positive $\beta_{1}<\beta$ :

$$
\begin{aligned}
\log m(\sigma+\alpha) & =\log m(\sigma+\alpha / 2)+\int_{\sigma+\alpha / 2}^{\sigma+\alpha} \lambda_{n(x)} d x \geqq(\alpha / 2) \lambda_{n(\sigma+\alpha / 2)} \\
& \geqq(\alpha / 2) \beta_{1} n(\sigma+\alpha / 2) .
\end{aligned}
$$

Therefore, applying Theorem 1 with $\delta(\sigma)=\alpha / 2$, we obtain

$$
M(\sigma) \leqq\left\{1+2\left(\alpha \beta_{1}\right)^{-1} \log m(\sigma+\alpha)+1 /\left[\exp \left(\beta_{1} \alpha / 2\right)-1\right]\right\} m(\sigma)
$$


and consequently for every $\epsilon>0$ there exists a number $\sigma(\epsilon)$ such that $\sigma \geqq \sigma(\epsilon)$ implies

$$
\log M(\sigma) \leqq(1+\epsilon) \log \log m(\sigma+\alpha)+\log m(\sigma)
$$

and obviously

$$
\log M(\sigma) \leqq(1+\epsilon) \log \log M(\sigma+\alpha)+\log m(\sigma) .
$$

By division of (11) or (12) by $\log m(\sigma)$ or by $\log M(\sigma)$, and using (9) or (10), respectively, we obtain

$$
\log M(\sigma) / \log m(\sigma) \leqq o(\sigma)+1
$$

or

$$
1 \leqq o(\sigma)+\log m(\sigma) / \log M(\sigma) .
$$

Every one of these inequalities combined with (3) implies

$$
\lim _{\sigma=\infty} \log M(\sigma) / \log m(\sigma)=1 \text {. }
$$

Theorem 2 generalises the result given by San Juan in the case of Taylor series [9, p. 134].

An obvious consequence of Theorem 2 is that if (8) holds, then conditions (9) and (10) are equivalent.

\section{REFERENCES}

1. J. F. Ritt, On certain points of the theory of Dirichlet series, Amer. J. Math. vol. 50 (1952) pp. 73-86.

2. G. Doetsch, Über die Obere Grenze des absoluten Betrages einer analytischen Funktion auf Geraden, Math. Z. vol. 8 (1920) pp. 237-240.

3. S. Mandelbrojt, Séries de Fourier et classes quasi-analytiques de fonctions, Paris, Gauthier-Villars, 1935.

4. - Dirichlet series, The Rice Institute Pamphlet, vol. 31, no. 4, October 1944, pp. 159-272.

5. A. G. Azpeitia, El orden precisado en las funciones enteras, Memorias de Matemáticas del Instituto Jorge Juan, no. 15, Consejo Superior de Investigaciones Cientificas, Madrid, 1955.

6. K. Sugimura, Übertragung einiger Satze aus der Theorie der ganzen Funktionen auf Dirichletsche Reihen, Math. Z. vol. 29 (1928-1929) pp. 264-277.

7. Q. I. Rahman, On the maximum modulus and the coefficients of an entire Dirichlet series, Tôhoku Math. J. (2) vol. 8 (1956) pp. 108-113.

8. G. Valiron, Lectures on the general theory of integral functions, New York, Chelsea, 1949.

9. R. San Juan, Los fundamentos de una teoría general de series divergentes, Rev. Acad. Ci. Madrid vol. 45 (1951) pp. 121-149.

UNIVERSITY OF MASSACHUSETTS 